\title{
El protocolo de curl nórdico y sus efectos en jugadores de fútbol. Una revisión narrativa
}

\section{The nordic hamstring exercise protocol and its effects on soccer Players. A narrative review}

\author{
Alma Rico-González (iD); Arely G. Morales-Hernández ${ }^{1}$ iD
}

'Universidad Autónoma de Querétaro; México. almagon17@hotmail.com; arelymorales@uaq.mx

Cómo citar: Rico-González, A.; Morales-Hernández, A.G. 2021. El protocolo de curl nórdico y sus efectos en jugadores de fútbol. Una revisión narrativa. Rev. Digit. Act. Fis. Deport. 7(2):e1905. http://doi.org/10.31910/ rdafd.v7.n2.2021.1905

Artículo de acceso abierto publicado por Revista Digital: Actividad Física y Deporte, bajo una licencia Creative Commons CC BY-NC 4.0

Publicación oficial de la Universidad de Ciencias Aplicadas y Ambientales U.D.C.A, Institución de Educación Superior Acreditada de Alta Calidad por el Ministerio de Educación Nacional.

Recibido: febrero 17 de 2021 Aceptado: marzo 29 de 2021 Editado por: Álvaro José Gracia Díaz

\section{RESUMEN}

Introducción: El fútbol es un deporte de masas, que tiene millones de practicantes y fans en todo el mundo. La lesión de isquiotibiales es la más frecuente en el soccer. El curl Nórdico es un ejercicio excéntrico que es fácil de practicar y no necesita ningún accesorio para realizarse, lo que lo convierte en ideal para complementar el entrenamiento de los jugadores de fútbol y, al mismo tiempo, disminuir el riesgo de lesión. Objetivo: Realizar una revisión narrativa para establecer el efecto del protocolo de Curl Nórdico en jugadores de fútbol. Métodos: La revisión de la literatura fue realizada en tres fases. La primera consistió en la búsqueda electrónica en las bases de datos de PubMed/ Medline, Clinical Key y Scielo, para identificar los artículos relevantes publicados, entre el 2001-2019; en la segunda fase, los títulos y los resúmenes de los resultados obtenidos fueron analizados y, por último, la lectura de todos los artículos potencialmente elegibles y separarlos acorde con su temática. Resultados: 115 artículos fueron encontrados, de los cuales, 40 cumplieron con el criterio de selección. Conclusión: Con este protocolo, se genera una ganancia de fuerza, que ha arrojado evidencia para incrementar los gestos del sprint y el cambio de dirección (que son esenciales en el soccer) y también para disminuir el riesgo de lesión.

Palabras clave: Ejercicio de curl nórdico; Fuerza excéntrica; Jugadores de fútbol; Lesiones.

\section{ABSTRACT}

Background: Soccer is a mass sport that has millions of practitioners and fans all over the world. Hamstring injuries are the most common muscle injuries in soccer. The Nordic curl is an eccentric exercise that is easy to perform and does not need any accessory to be carried out. This makes it ideal to complement the training of soccer players and at the same time reduce the risk of injury. Purpose: To establish the effect of the Nordic hamstring exercise protocol in soccer players. Methods: The literature review was made in three phases. The first phase consisted of an electronic database search of PubMed, Medline, Clinical Key and Scielo to identify relevant articles published between 2001-2019. In the second phase, the titles and summaries of the results obtained were 
analyzed. And finally, the third phase consisted of reading all the articles potentially eligible and separate them according to their thematic. Results: 115 articles were found of which only 40 met with the selection criteria. Conclusion: There is a gain in strength with this protocol, which has led to evidence of increased sprinting and change of direction (gestures that are essential in soccer) and also a decrease in the risk of injury.

Keywords: Nordic hamstring exercise; Eccentric strength; Soccer players; Injuries.

\section{INTRODUCCIÓN}

El fútbol es un deporte de masas que tiene millones de practicantes y de aficionados en todo el mundo. De hecho, es el deporte más popular y jugado sin excepción. El fútbol tiene una serie de características complejas, debido a que es un deporte colectivo, de cooperación y oposición, que se desarrolla, a través de habilidades abiertas (Gil et al. 2018). Asimismo, debido a estas y algunas otras características, que son las razones, por las cuales, es difícil aislar y definir cuáles son los factores que determinan el éxito o el máximo rendimiento (Benítez Sillero et al. 2015). Los practicantes de fútbol, especialmente los aficionados y los más jóvenes, buscan el fortalecimiento muscular en el gimnasio, para complementar sus sesiones de entrenamiento o como el único elemento de su entrenamiento.

Estudios recientes demuestran que los requisitos físicos y fisiológicos en el fútbol, se caracterizan por la presencia de muchos movimientos de alta intensidad, como saltos, aceleraciones, cambios de dirección y sprints repetidos (Reilly et al. 2000; Haff et al. 2001). Estas acciones de alta intensidad, se deben tener en cuenta, a la hora de programar un entrenamiento de fútbol (Raya-González et al. 2017). La fuerza muscular juega un papel importante en la prevención de lesiones, a través de la estabilización dinámica de la articulación. Además, de la fuerza muscular individual, otro factor en la prevención de lesiones es la relación de fuerza entre los músculos agonistas y antagonistas (Navandar et al. 2018). La relación de fuerza que ha recibido mayor importancia en la literatura es la que está entre los isquiotibiales y el cuádriceps ( $\mathrm{H}: \mathrm{Q}$ ). Hay una buena razón para esto; si la fuerza del cuádriceps supera significativamente la fuerza de los isquiotibiales, entonces, el ligamento cruzado anterior (ACL) y los isquiotibiales serán más susceptibles a lesiones (Ishøi et al. 2018).

Además, con la ayuda de los isquiotibiales, el LCA estabiliza la rodilla, evitando el desplazamiento anterior de la tibia con respecto al fémur. Este desplazamiento anterior puede ocurrir durante los aterrizajes de saltos y los cambios repentinos de dirección (movimientos típicos de los jugadores de fútbol). Cuando los cuádriceps superan en fuerza a los isquiotibiales, un desplazamiento anterior se genera en la articulación de la rodilla; ésto, aunado a los movimientos dinámicos que suceden en el fútbol, provoca que el LCA experimente altas fuerzas y desencadene un desgarre del mismo (Monajati et al. 2017). El entrenamiento de fuerza es la forma más adecuada de corregir este problema, pero muchos ejercicios, especialmente las sentadillas, suelen implicar el cuádriceps, a un nivel más alto que los isquiotibiales, lo que causa una disminución en la relación de fuerza $H$ : $Q$. Esta relación $H$ : $Q$ se ha utilizado como técnica preventiva para lesiones de rodilla e isquiotibiales. Las personas, con una relación $\mathrm{H}$ : $\mathrm{Q}$ baja, están predispuestas a un mayor riesgo de lesiones. La implementación de programas de fortalecimiento, antes de que comiencen los períodos de competición del atleta, aumenta su rendimiento físico y reduce el riesgo de lesiones (Ardern et al. 2015). Las lesiones por tensión en la musculatura de miembros inferiores forman parte de, aproximadamente, $1 / 3$ de todas las lesiones en el fútbol profesional y los desgarres en los músculos isquiotibiales, son los más comunes. Los programas de entrenamiento de fuerza con sobrecarga excéntrica han tenido una presencia creciente en la periodización del entrenamiento en el fútbol (RayaGonzález et al. 2017). Estos ejercicios pueden traer mayores beneficios en términos de fuerza, movilidad y prevención de lesiones (Serner et al. 2019).

El ejercicio de curl nórdico (NHE) es un ejercicio excéntrico, que ha demostrado ser una técnica eficaz, para el control dinámico y la fuerza excéntrica en los isquiotibiales. Este ejercicio desarrolla un torque máximo de fuerza excéntrica en los isquiotibiales en comparación con el curl de bíceps femoral común. Estudios previos en futbolistas profesionales masculinos han demostrado que la implementación del curl nórdico en el entrenamiento regular reduce la incidencia de lesiones en los isquiotibiales, entre un 65 y un 70\% (Van Der Horst et al. 2015). Siddle 
et al. (2019) concluyeron en un estudio, que un programa de intervención con el NHE de 6 semanas, mostró mejoras significativas inmediatamente, post intervención, en la fuerza excéntrica de los isquiotibiales y un mejor rendimiento en la prueba de sprint de 10 metros y en la velocidad del cambio de dirección (COD) y se mantuvo después de un período de desentrenamiento de 3 semanas (Tabla
1). En consecuencia, se puede implementar el NHE, como ejercicio de prevención de lesiones y mejorar, simultáneamente, el rendimiento funcional. Fernández-Gonzalo et al. (2016) concluyeron que la estimulación mecánica de este ejercicio excéntrico parecía modificar la arquitectura muscular de la cabeza larga del bíceps femoral, aumentando la longitud del fascículo (FL).

Tabla 1. Protocolo de curl nórdico Van Der Horst et al. (2015).

\begin{tabular}{|c|c|c|}
\hline Semana & Sesión por semana & Sets y repeticiones \\
\hline 1 & 1 & $2 \times 5$ \\
\hline 2 & 2 & $2 \times 6$ \\
\hline 3 & 3 & $3 \times 6-8$ \\
\hline 4 & 3 & $3 \times 8-10$ \\
\hline $5-10$ & 3 & 3 sets, $12-10-8$ reps \\
\hline $11-13$ & 1 & 3 sets, $12-10-8$ reps \\
\hline
\end{tabular}

La razón para elegir el NHE es por su simplicidad y su facilidad de replicación; por lo tanto, se puede aplicar fácilmente a cualquier atleta, de manera individual o colectiva, en programas de prevención o rehabilitación (Barbacena \& Norina, 2015). El objetivo principal de esta revisión narrativa es recolectar todos los estudios existentes sobre el protocolo de curl nórdico y sus efectos en futbolistas.

\section{MATERIALES Y MÉTODOS}

El presente artículo es una revisión exhaustiva narrativa de los conocimientos existentes sobre el protocolo de curl nórdico y su eficacia en los jugadores de fútbol. La revisión de la literatura, se realizó en tres fases. La primera fue una búsqueda electrónica de bases de datos, como PubMed/ Medline, Clinical Key y Scielo, para identificar los artículos relevantes, publicados entre 1994-2019. La búsqueda de palabras clave en estos artículos y los términos clave de búsqueda incluidos fueron soccer AND Nordic exercises, OR Nordic hamstring exercise AND Nordic hamstring protocol OR eccentric strength OR hamstring injuries y combinaciones de ellos. También, se tomaron en cuenta listas de referencias de estudios y artículos identificados que citaban los estudios primarios, con títulos que incluyeran las palabras clave de búsqueda (125 artículos).

Después, en la segunda fase, se revisaron títulos y resúmenes de los resultados obtenidos. Como criterios de inclusión, solo se consideraron los artículos que utilizaran a los jugadores de fútbol o atletas, como población de estudio. Las variables de estudio fueron: relación funcional $\mathrm{H}$ : Q, ejercicio excéntrico, lesiones en los isquiotibiales, ejercicio de curl nórdico, protocolo de curl nórdico, sprint y cambio de dirección. Artículos únicamente pertenecientes a revistas indexadas en idiomas en español, inglés o francés, sin ningún tipo de restricción temporal. Se excluyeron artículos, donde la población de estudio no fueran atletas o jugadores de fútbol (68 artículos).

La tercera fase consistió en la lectura de todos los artículos potencialmente elegibles y se agruparon, según su temática y términos de búsqueda propuestos. Durante esta fase, los artículos fueron sometidos a revisión por parte de dos revisores, para determinar los artículos finales (50 artículos).

\section{RESULTADOS Y DISCUSIÓN}

Relación funcional $\mathrm{H}: \mathrm{Q}$. El fútbol es un deporte asociado con una alta prevalencia de lesiones, tanto a nivel profesional como amateur (Azubuike \& Okojie, 2009). La fuerza muscular juega un papel importante en la prevención de lesiones, a través de la estabilización dinámica de la articulación (Gissis et al. 2006). Los desequilibrios de fuerza, se han asociado con el riesgo de lesiones en el deporte; además de la fuerza muscular individual, otro factor en la prevención de lesiones, es la relación de fuerza entre los músculos agonistas y antagonistas. Un desequilibrio muscular, comúnmente sometido a pruebas, es la relación de fuerza de isquiotibialescuádriceps (H: Q). 
La relación $\mathrm{H}$ : $\mathrm{Q}$, se calcula dividiendo el pico de toque de la contracción concéntrica máxima de la flexión de la pierna y el pico de torque de la contracción concéntrica de la extensión de pierna a la misma velocidad angular (Kannus, 1994). Esta proporción indica una fuerte comparación entre los lados opuestos de la musculatura (Holcomb et al. 2007).

La evaluación isocinética es uno de los métodos más discutidos dentro de la literatura actual y es utilizado para evaluar el riesgo de lesión muscular, utilizando la relación $\mathrm{H}: \mathrm{Q}$, como indicador clave (McCall et al. 2015; Fousekis et al. 2011). Heiser et al. (1984) evaluaron la fuerza, mediante el uso de un dinamómetro isocinético, para determinar la prevención de lesiones en los isquiotibiales. Una relación $\mathrm{H}$ : $\mathrm{Q}$ de menos de 0,6 y una asimetría flexora del 5\%, representaría un factor de riesgo para la aparición de daño muscular en los isquiotibiales. Así, el uso del fortalecimiento, como corrección de estos parámetros, reduciría la tasa de lesiones en los isquiotibiales, del 7,6 al 1\% y la tasa de recurrencia del 31,7 al $0 \%$, en el fútbol americano.

Kaminski et al. (1998) encontraron que, en un grupo de estudiantes universitarios no entrenados, la implementación de un entrenamiento de fuerza excéntrico era más eficaz que el entrenamiento de fuerza concéntrica en el pico isocinético de la fuerza excéntrica en los isquiotibiales, después de implementar 6 semanas de entrenamiento (dos sesiones por semana), en una máquina de curl de pierna.

Ejercicios excéntricos. El fortalecimiento excéntrico es el más olvidado, cuando se trata de entrenamiento de fuerza; sin embargo, este tipo de fortalecimiento es el que puede traer los mayores beneficios en fuerza, movilidad y prevención de lesiones (De la Torre, 2018). Los programas de fuerza con sobrecarga excéntrica han tenido una presencia creciente en la periodización de entrenamiento en el fútbol. Representan un factor de riesgo modificable para las lesiones por tensión en los isquiotibiales. Es importante mencionar que los músculos isquiotibiales consisten, principalmente, en fibras tipo I, que tardan más en contraerse y ayudan a continuar con una actividad después de largos períodos de tiempo (McHugh, 2003). Es por eso, que responden mejor a un alto número de repeticiones lentas y ejercicios excéntricos. En estudios anteriores, se demostró que los programas de entrenamiento excéntricos parecen producir hipertrofia músculo esquelético temprana (Siddle et al. 2019).
Prevención de lesiones de isquiotibiales. Las lesiones en el fútbol representan el deterioro más común para los jugadores en los entrenamientos y partidos (Dvorak et al. 2011). Las lesiones en los isquiotibiales son las lesiones más frecuentes por no contacto en los deportes de conjunto (Brooks et al. 2006; Feeley et al. 2008; Ekstrand et al. 2016). Una lesión en los isquiotibiales, se define como cualquier queja física aguda de la región posterior del muslo, sufrida durante un partido de fútbol o entrenamiento (Petersen \& Hölmich, 2005). El bíceps femoral (BF) (y, en menor medida, el semitendinoso -ST-) es comúnmente sometido a lesiones estructurales o funcionales (Opar et al. 2012). Estos músculos son más propensos a sufrir lesiones durante aceleraciones explosivas de carrera y de actividades que impliquen patadas, debido a las altas exigencias biomecánicas colocadas en la unidad músculo-tendón a lo largo de las fases de balanceo, durante la carrera y al patear (Schuermans et al. 2016).

La literatura científica describe dos mecanismos de lesión en los isquiotibiales. El primero ocurre cuando el músculo está sometido a posiciones articulares extremas $y$, el segundo, en una carrera de alta velocidad. Durante la fase de oscilación terminal del ciclo de marcha, los isquiotibiales son necesarios para desacelerar excéntricamente la extensión de la rodilla y la flexión de la cadera antes de soportar el peso. Este estrés excéntrico elevado contribuye a las altas tasas de lesiones en los isquiotibiales durante cualquier movimiento de carrera (Alonso-Fernández et al. 2018).

Por lo tanto, las estrategias preventivas de entrenamiento de fuerza también podrían incluir ejercicios, donde los músculos se activan durante la fase excéntrica para una respuesta muscular adecuada (De Hoyo et al. 2015). En un estudio epidemiológico de lesiones en los isquiotibiales, Woods et al. (2004) reportaron todas las lesiones de 91 clubes de fútbol profesional, durante más de dos temporadas. Se utilizó un cuestionario específico de auditoría de lesiones, junto con un formulario semanal, que documentaba el estado actual de lesiones de cada club. Esto representó un gran número de variables en el diagnóstico, las técnicas de capacitación y el manejo médico, pero, al menos, sugiere que estas lesiones se pueden prevenir y controlar (Woods et al. 2004).

Ejercicio de Hamstring Nórdico. El ejercicio de curl nórdico (NHE) es un ejercicio de parejas. El atleta 
comienza en una posición de hincado con el torso de rodillas para arriba totalmente recto; un compañero de entrenamiento debe aplicar presión en los tobillos del atleta, para asegurarse que los pies permanezcan en contacto con el suelo durante todo el movimiento. El atleta, entonces, intenta resistirse a un movimiento hacia adelante, usando sus músculos isquiotibiales, para maximizar la carga en la fase excéntrica. El atleta rompe la caída hacia adelante durante el mayor tiempo posible, usando los isquiotibiales; al final, utiliza sus brazos y manos para amortiguar la caída, dejando que el pecho toque la superficie e, inmediatamente, vuelve a la posición inicial, empujando con sus manos, para minimizar la carga en la fase concéntrica (Figura 1) (Barbacena \& Norina, 2015).

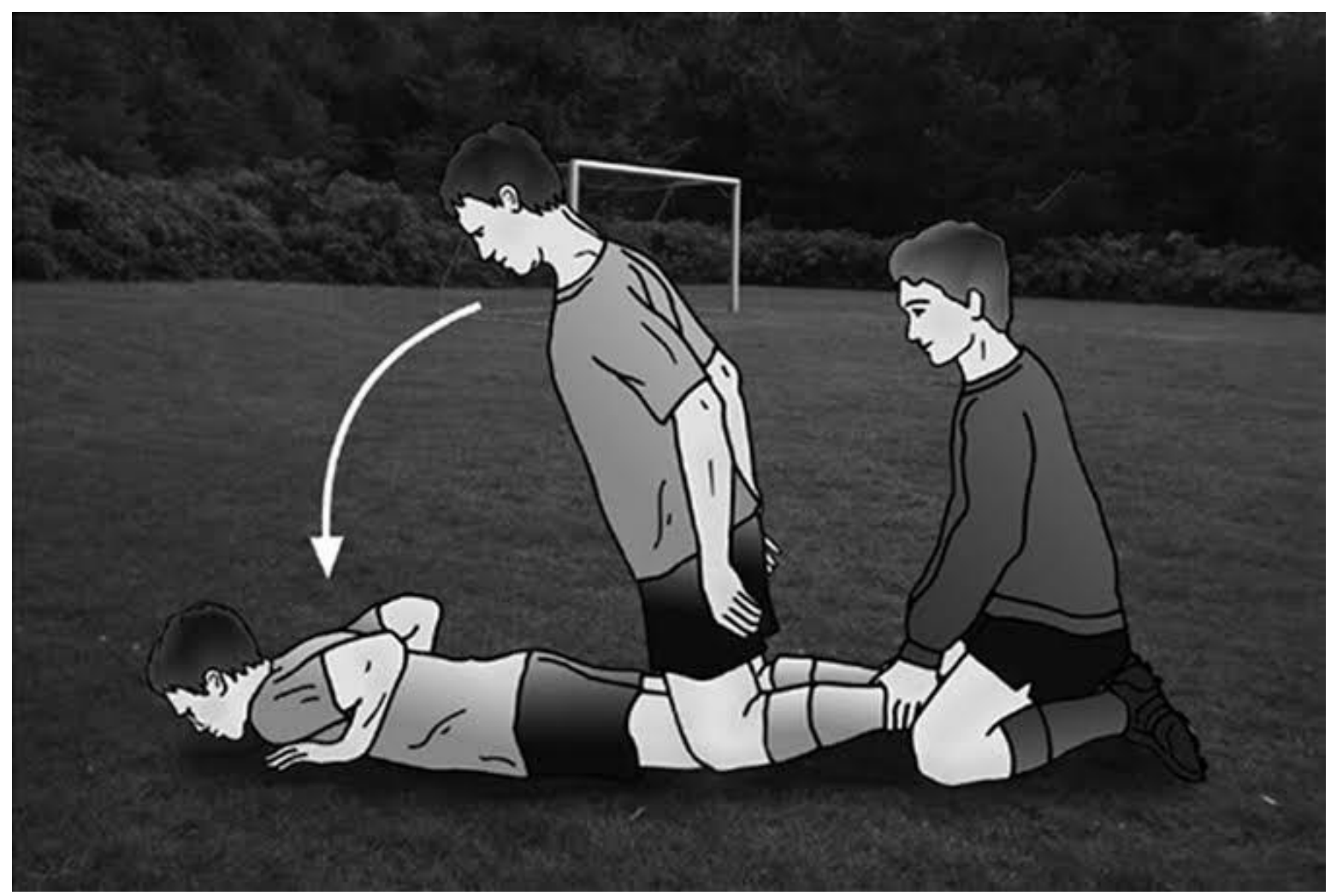

Figura 1. Ejercicio de curl nórdico, Petersen et al. 2011.

Seymor e et al. (2017) discutieron en su estudio los efectos del entrenamiento de fuerza excéntrica con el $\mathrm{NHE}$, en la arquitectura muscular de los isquiotibiales, en la rigidez y en la fuerza, pero a pesar de los aumentos significativos en el crecimiento muscular, su grupo experimental no aumentó significativamente la fuerza máxima excéntrica de los isquiotibiales $(p=0,563)$. Concluyeron, que el NHE es un buen ejercicio de entrenamiento para el aumento muscular y el volumen muscular, pero no para la longitud del fascículo.

Protocolo de curl nórdico para isquiotibiales. La prevención de lesiones nuevas y recurrentes de los isquiotibiales en el fútbol amateur y sub-élite, se ha logrado de manera efectiva en varias pruebas, mediante la implementación en la pretemporada del protocolo de curl nórdico. Este es un protocolo de fortalecimiento excéntrico para isquiotibiales, basado en el ejercicio de curl nórdico (Petersen et al. 2011; Van Der Horst et al. 2015). El protocolo establecido para la aplicación del curl nórdico comienza con una fase de incremento (semana 1 a 5) y continúa con una fase de mantenimiento (semana 6 a 13) (Schache, 2012).

Los efectos del protocolo de curl nórdico, se consideran esenciales para el rendimiento del sprint (Morín et al. 2015) y los estudios que utilizaron otros entrenadores, para aumentar la fuerza excéntrica de los isquiotibiales, también han reportado mejoras en la fuerza explosiva (Mancera-Soto et al. 2016), en el salto (Hasson et al. 2004) y en el rendimiento del sprint (Askling et al. 2003).

Velocidad y cambio de dirección. Siddle et al. (2019), en un estudio en el que se implementó un programa de intervención con el NHE de 6 semanas, reportaron 
una mejoría significativa en el cambio de dirección (COD) $(-0,12$ s vs 0,20 s; diferencia promedio- $0,332 \mathrm{~s}, \mathrm{P}$ $=0,003, d=2,17)$ y en la prueba de $10 \mathrm{~m}$ sprint $(-0,06 \mathrm{~s}$ vs $0,05 \mathrm{~s}$; diferencia promedio $-0,115 \mathrm{~s}, \mathrm{P}=0,024, \mathrm{~d}=$ 178); al mismo tiempo, observaron mejoras en las medidas de fuerza excéntrica de los isquiotibiales $\left(31,81 \mathrm{Nm}^{-1}\right.$ vs $6,44 \mathrm{Nm}^{-1}$; diferencia promedio $\left(29,46 m^{-1}, P=0,001, d=2,55\right)$; post intervención (Ishøi et al. 2018) obtuvieron mejoras similares, utilizando varias medidas de fuerza excéntrica en los isquiotibiales, siguiendo un programa de 10 semanas, aplicando el NHE.

Sun et al. (2015) estudiaron las condiciones de carga durante el máximo esfuerzo en el sprint. Hipotetizaron, que habría un gran riesgo de tensión en los isquiotibiales durante la fase de despegue y las fases de oscilación, en comparación con otras fases del ciclo de marcha en el sprint. Concluyeron, que durante las fases de despegue y de oscilación, los torques largos pasivos en las articulaciones de rodilla y de cadera actuaron para alargar los músculos isquiotibiales. Como resultado, durante el sprint - la locomoción de alta velocidad, los músculos isquiotibiales pueden ser más susceptibles a un alto riesgo de lesión por tensión, durante estas dos fases.

Ensu estudio Yu etal.(2008) analizaron los mecanismos de lesiones por tensión muscular en los isquiotibiales durante el sprint, mediante la investigación de la cinemática músculo-tendón de los isquiotibiales y la activación muscular. Llegaron a la conclusión, que el potencial de lesión por tensión muscular en los isquiotibiales, se presenta durante la fase de despegue, así como durante las fases de balanceo del sprint. Asimismo, Thelen et al. (2005) graficó la cinemática de los músculos isquiotibiales durante el sprint, para proporcionar datos científicos y así comprender mejor los mecanismos de lesiones y las diferencias en las tasas de lesiones entre los músculos. Sus hallazgos corresponden a que las diferencias intermusculares en el brazo, en el momento de los isquiotibiales sobre la cadera y la rodilla, pueden ser un factor que contribuye al aumento de riesgo de lesión por tensión en los isquiotibiales, específicamente, en el músculo BF.

En cuanto a los estudios en los que se compara el protocolo de curl nórdico contra el curl tradicional de bíceps femoral (Barbacena \& Norina, 2015) determinó, después de un entrenamiento de 10 semanas, que el protocolo de curl nórdicos es más eficaz, para desarrollar la fuerza de los isquiotibiales en los jugadores de fútbol, provocando un aumento del $11 \%$ en el torque, en comparación con un aumento del $7 \%$, utilizando el curl tradicional del bíceps ( $\mathrm{P}=$ $0,001)$.

En este sentido, los resultados fueron similares a los que (Siddle et al. 2019), en su ensayo clínico aleatorizado, al que se realizó con 2 mediciones en atletas. La primera, después de la aplicación de 6 semanas de intervención, con el curl nórdico y, la segunda, después de 3 semanas de desentrenamiento. Los hallazgos clave demostraron que inmediatamente post- intervención mejoró, de manera significativa, el rendimiento del COD y de la prueba de sprint de 10m; también, se observaron mejoras en las medidas de fuerza excéntrica de los isquiotibiales. Las mejoras del rendimiento, se mantuvieron después de un período de desentrenamiento para el COD y sprint, pero no en la fuerza excéntrica de los isquiotibiales. Estudios previos sobre el rendimiento del sprint en jugadores élite de fútbol masculinos han demostrado una diferencia en los tiempos máximos de sprint de $10 \mathrm{~m}$, entre la parte superior e inferior del percentil $25^{\circ}$, de 0,08seg (Haugen et al. 2014). Otros estudios, también han utilizado el mismo período de desentrenamiento en sus investigaciones; un ejemplo es AlonsoFernández et al. (2018), quienes desarrollaron un estudio para determinar las alteraciones estructurales del músculo, utilizando una ecografía en la cabeza larga del bíceps femoral, después de implementar el protocolo de curl nórdico. También, consideraron 3 semanas de desentrenamiento, como los estudios anteriores, realizando 3 mediciones. El primero, en la semana 1, el segundo en la semana 9 y el último en la 13a semana (después de 3 semanas de desentrenamiento). En la segunda medición, un aumento en la longitud ( $t=-7,73, d=2,28, P<.001)$ y grosor $(t=-5,23, d=1.54, P<.001$ ) del músculo y una disminución del ángulo de penación ( $t=7,81, d=2,3$, $\mathrm{P}<.001)$ fueron reportados. En la tercera medición, se observó una disminución de la longitud ( $t=6,07$, $d=1,79, P<.001)$, una disminución en el grosor $(t=$ $3,64, d=1,07, P<.05)$ y un aumento en el ángulo de penación ( $t=-4,63, d=136, P<.001)$. Otros estudios sugieren que los efectos más largos han sido vistos cuando el entrenamiento de spring se lleva a cabo en la temporada baja o antes de la pretemporada (Tønnessen etal.2011). Con esto, se puede determinar que el protocolo de curl nórdico tiene impactos 
estructurales en el músculo. El estudio, previamente mencionado, puede confirmar la hipótesis sobre que el ejercicio de curl nórdico aumenta la fuerza de los isquiotibiales, obteniendo una disminución en los factores de riesgo, que provocan las lesiones en el fútbol.

Por el contrario, en un estudio reportado por Bourne et al. 2017, donde evaluaron los cambios de longitud en el fascículo de la cabeza larga del bíceps femoral (BFLH) y el tamaño de los isquiotibiales, siguiendo un entrenamiento de 10 semanas, utilizando el ejercicio de curl y el ejercicio de extensión de cadera; los hallazgos fueron que la extensión de cadera activaba selectivamente los isquiotibiales (diferencia promedio $=0,7,95 \% \mathrm{Cl} 0,6$ a $0,9, p<0,001)$ y el ejercicio de curl nórdico reclutaba, preferentemente, al semitendinoso (diferencia promedio $=0,51,95 \% \mathrm{Cl}$ 0,39 a 0,64, p<0,001) y que el volumen del bíceps femoral aumentaba más con la extensión de la cadera que con el ejercicio de curl nórdico y el grupo control (diferencia promedio $=0,42,95 \% \mathrm{Cl} 0,24$ a 0,62, $p<0,001)$. Con esto, se puede asumir que el ejercicio de extensión de cadera puede ser más efectivo en promover la hipertrofia en el BFLH.

\section{CONCLUSIONES}

Por lo tanto, se concluye que se necesitan más investigaciones para poder determinar cuánto tiempo después de la aplicación del protocolo de curl nórdico los efectos continúan. Esta información ayudaría a los entrenadores a determinar en qué momento exacto de su macrociclo deben implementarlo, para que, en el período de competición, sus atletas puedan obtener el efecto máximo de este protocolo y así disminuir el riesgo de lesión y, al mismo tiempo, aumentar sus capacidades físicas, como deportistas.

Con el fin de mejorar y de optimizar el rendimiento de los jugadores de fútbol, se han utilizado diferentes programas de entrenamiento de fuerza, que consisten en un gran número de ejercicios diferentes. Debido a estudios anteriores, se sabe que los programas de fortalecimiento excéntrico reducen el riesgo de lesiones y producen una hipertrofia temprana. El curl nórdico es un ejercicio excéntrico, que es fácil de realizar y no necesita ningún equipo adicional y lo hace ideal para complementar el entrenamiento de los futbolistas y reducir el riesgo de lesión. Es esencial, en el campo deportivo, identificar cómo prevenir, adecuadamente, una de las lesiones más frecuentes en el fútbol: la lesión de isquiotibiales y la lesión de ligamento cruzado anterior. El curl nórdico es un ejercicio simple y rápido con muchos beneficios. Los entrenadores deben ser capaces de incluir este ejercicio como parte de su entrenamiento. Asimismo, un dato muy importante que producen los estudios existentes es que, uno de los beneficios del uso del protocolo de curl nórdico es permitir una periodización progresiva, provocando que los atletas se puedan adaptar al ejercicio excéntrico.

Los estudios determinan que, con este protocolo, hay una ganancia de fuerza que también ha llevado a evidenciar un aumento en los gestos del sprint y el gesto del cambio de dirección, que son esenciales en el fútbol.

Agradecimientos. A la Facultad de Enfermería de la Universidad Autónoma de Querétaro. Financiación: No se recibió apoyo financiero para la preparación de este manuscrito. Declaración de interés: Los autores no informaron de ningún conflicto de intereses.

\section{REFERENCIAS}

1. ALONSO-FERNÁNDEZ, D.; DOCAMPO-BLANCO, P.; MARTÍNEZ-FERNÁNDEZ, J. 2018. Changes in muscle architecture of biceps femoris induced by eccentric strength training with nordic hamstring exercise. Scandinavian Journal of Medicine \& Science in Sports. 28(1):88-94. https:// doi.org/10.1111/sms.12877

2. ARDERN, C.; PIZZARI, T.; WOLLÍN, M.; WEBSTER, K. 2015. Hamstrings strenghth imbalance in professional football (soccer) players in Australia.TheJournalofStrength\&Conditioning Research. 29(4):997-1002. https://doi. org/10.1519/jsc.0000000000000747

3. ASKLING, C.; KARLSSON, J.; THORSTENSSON, A. 2003. Hamstring injury occurence in elite soccer players after preseason training with eccentric loading. Scandinavian Journal of Medicine and Science in Sports. 13(4):244$250 . \quad$ https://doi.org/10.1034/ j.1600-0838.2003.00312.x

4. AZUBUIKE, S.; OKOJIE, O. 2009. An epidemiological study of football (soccer) injuries in Benin City, Nigeria. British Journal of Sports Medicine. 43(5):382-386. https:// doi.org/10.1136/bjsm.2008.051565 
5. BARBACENA, L.; NORINA, R.; 2015. Muscle strain injuries in sports - A 10-week randomised trial comparing eccentric vs. concentric hamstring strength in well trained soccer players. Intersection. 12(1):16-22.

6. BENÍTEZ SILLERO, B.; SILVA-GRIGOLETTO, D.; HERRERA, M.; MORENTE MONTERO, A.; GUILLÉN DEL CASTILLO, M. 2015. Capacidades físicas en jugadores de fútbol formativo de un club profesional. Revista Internacional de Medicina y Ciencias de la Actividad Física y el Deporte. 15(58):289-307. https:// doi.org/10.15366/rimcafd2015.58.006

7. BOURNE, M.; WILLIAMS, M.; OPAR, D.; AL NAJJAR, A.; KERR, G.; SHIELD, A. 2017. Impact of exercise selection on hamstring muscle activation. British Journal of Sports Medicine. 51(13):1021-1028. https:// doi.org/10.1136/bjsports-2015-095739

8. BROOKS, J.; FULLER, C.; KEMP, S.; REDDIN, D. 2006. Incidence, risk, and prevention of hamstring muscle injuries in professional rugby union. American Journal of Sports Medicine. 34(8):1297-1306. https://doi. org/10.1177/0363546505286022

9. DE HOYO, M.; POZZO, M.; SAÑUDO, B.; CARRASCO, L.; GONZALO-SKOK, O.; DOMÍNGUEZ-COBO, S.; MORÁN-CAMACHO, E. 2015. Effects of a 10-week in-season eccentric-overload training program on muscle-injury prevention and performance in junior elite soccer players. International Journal of Sports Physiology and Performance. 10(1):46-52. http://dx.doi.org/10.1123/ijspp.2013-0547

10. DE LA TORRE, G. 2018. Salud De Hierro. Traitlon. 78-81. Disponible desde Internet en: https://centropronaf.com/ wp-content/uploads/2016/09/TRI-4478-81-ENTRENAMIENTO-EXCENTRICO. pdf (con acceso el 23/04/2020)

11.DVORAK,J.;JUNGE,A.;DERMAN,W.;SCHWELLNUS, M. 2011. Injuries and illnesses of football players during the 2010 FIFA World Cup. British Journal of Sports Medicine. 45(8):626-630. https://doi.org/10.1136/bjsm.2010.079905
12. EKSTRAND, J.; WALDEN, M.; HÄGGLUND, M. 2016. Hamstring injuries have increased by $4 \%$ annually in men's professional football, since 2001: A 13-year longitudinal analysis of the UEFA Elite Club injury study. British Journal of Sports Medicine. 50(12):731-737. http:// dx.doi.org/10.1136/bjsports-2015-095359

13. FEELEY, B.; KENNELLY, S.; BARNES, R.; MULLER, M.; KELLY, B.; RODEO, S.; WARREN, R. 2008. Epidemiology of national football league training camp injuries from 1998 to 2007. American Journal of Sports Medicine. 36(8):1597-1603. https:// doi.org/10.1177/0363546508316021

14. FERNANDEZ-GONZALO, R.; TESCH, P.A.; LINNEHAN, R.M.; KREIDER, R.B.; DI SALVO, V.; SUAREZ-ARRONES, L.; ALOMAR, X.; MENDEZ-VILLANUEVA, A.; RODAS, G. 2016. Individual muscle use in hamstring exercises by soccer players assessed using functional MRI. International journal of sports medicine. 37(7):559-564. https://doi.org/10.1055/s-0042-100290

15. FOUSEKIS, K.; TSEPIS, E.; POULMEDIS, P.; ATHANASOPOULOS, A.; VAGENAS, G. 2011. Intrinsic risk factors of non-contact quadriceps and hamstring strains in soccer: A prospective study of 100 professional players. British Journal of Sports Medicine. 45(9):709-714. https://doi.org/10.1136/bjsm.2010.077560

16. GIL, S.; BARROSO, R.; CRIVOI DO CARMO, E.; LOTURCO, I.; KOBAL, R.; TRICOLI, V.; UGRINOWITSCH, C.; ROSCHEL, H. 2018. Effects of resisted sprint training on sprinting ability and change of direction speed in professional soccer players. Journal of Sports Sciences. 36(17):1923-1929. https://doi.org/10.1080/ 02640414.2018.1426346

17. GISSIS, I.; PAPADOPOULOS, C.; KALAPOTHARAKOS, V.; SOTIROPOULOS, A.; KOMSIS, G.; MANOLOPOULOS, E. 2006. Strength and speed characteristics of elite, subelite, and recreational young soccer players. Research in Sports Medicine. 14(3):205-214. https://doi. org/10.1080/15438620600854769 
18. hAFF, A.; WHITLEY, A.; POTTEIGER, J. 2001. A Brief Review: Explosive Exercises and Sports Performance. Strength and Conditioning Journal. 23(3):13-20.

19. HASSON, C.; DUGAN, E.; DOYLE, T.; HUMPHRIES, B.; NEWTON, R. 2004. Neuromechanical strategies employed to increase jump height during the initiation of the squat jump. Journal of Electromyography and Kinesiology. 14(4):515-521. https:// doi.org/10.1016/j.jelekin.2003.12.004

20. HAUGEN, T.; TøNNESSEN, E.; HISDAL, J.; SEILER, S. 2014. The Role and Development of Sprinting Speed in Soccer. International Journal of Sports Physiology and Performance. 9(3):432-441. https://doi.org/10.1123/ijspp.2013-0121

21. HEISER, T.M.; WEBER, J.; SULLIVAN, G.; CLARE, P.; JACOBS, R.R. 1984. Prophylaxis and management of hamstring muscle injuries in intercollegiate football players. American Journal of Sports Medicine 12(5):368-370. https://doi. org/10.1177/036354658401200506

22. HOLCOMB, W.; RUBLEY, M.; LEE, H.; GUADAGNOLI, M. 2007. Effect of Hamstring-Emphasized Resistance Training on Hamstring. Journal of Strength and Conditioning Research. 21(1):4147. https://doi.org/10.1519/r-18795.1

23. ISHØI, L.; HÖLMICH, P.; AAGAARD, P.; THORBORG, K.; BANDHHOLM, T.; SERNER, A. 2018. Effects of the Nordic Hamstring exercise on sprint capacity in male football players: a randomized controlled trial. Journal of Sports Sciences. 36(14):1663-1672. https:// doi.org/10.1080/02640414.2017.1409609

24. KAMINSKI, T.; WABBERSEN, C.; MURPHY, R. 1998. Concentric versus enhanced eccentric hamstring strength training: Clinical implications. Journal of Athletic Training. 33(3):216-221.

25. KANNUS, P. 1994. Isokinetic evaluation of muscular performance: implications for muscle testing and rehabilitation. International Journal of Sports Medicine. 15(1):11-18. https://doi.org/10.1055/s-2007-1021104
26. MANCERA-SOTO, É.; PÁEZ, A.; FÚQUENE, M.; AVELLANEDA, P.; CORTÉS, S.; QUICENO, C.; RAMOS-CABALLERO, D. 2016. Efectividad de un protocolo de entrenamiento nórdico sobre la fuerza explosiva en futbolistas del Club Deportivo La Equidad Seguros. Revista de La Facultad de Medicina. 64(3):17-24. http://dx.doi. org/10.15446/revfacmed.v64n3Sup.51061

27. MCCALL, A.; CARLIG, C.; DAVISON, M.; NEDELEC, M.; LE GALL, F.; BERTHOIN, S.; DUPONT, G. 2015. Injury risk factors, screening tests and preventative strategies: A systematic review of the evidence that underpins the perceptions and practices of 44 football (soccer) teams from various premier leagues. British Journal of Sports Medicine. 49(9):583-589. https:// doi.org/10.1136/bjsports-2014-094104

28. MCHUGH, M. 2003. Recent advances in the understanding of the repeated bout effect: The protective effect against muscle damage from a single bout of eccentric exercise. Scandinavian Journal of Medicine and Science in Sports. 13(2):88-97. https://doi. org/10.1034/j.1600-0838.2003.02477.x

29. MONAJATI, A.; LARUMBE-ZABALA, E.; GOSSSAMPSON, M.; NACLERIO, F. 2017. Analysis of the Hamstring Muscle Activation during two Injury Prevention Exercises. Journal of Human Kinetics. 60(1):29-37. https://doi.org/10.1515/hukin-2017-0105

30. MORÍN, J.; GIMÉNEZ, P.; EDOUARD, P.; ARNAL, P.; JIMÉNEZ-REYES, P.; SAMOZINO, P.; BRUGHELLI, M.; MENDIGUCHIA, J. 2015. Sprint acceleration mechanics: The major role of hamstrings in horizontal force production. Frontiers in Physiology. 6(11):1-14. https:// dx.doi.org/10.3389/fphys.2015.00404

31. NAVANDAR, A.; VEIGA, S.; TORRES, G.; CHORRO, D.; NAVARRO, E. 2018. A previous hamstring injury affects kicking mechanics in soccer players. The Journal of Sports Medicine and Physical Fitness. 58(12):1815-1822. https:// doi.org/10.23736/s0022-4707.18.07852-0 
32. OPAR, D.; WILLIAMS, M.; SHIELD, A. 2012. Hamstring strain injuries: Factors that Lead to injury and re-Injury. Sports Medicine. 42(3):209-226. https://doi. org/10.2165/11594800-000000000-00000

33. PETERSEN, J.; HÖLMICH, P. 2005. Evidence based prevention of hamstringinjuries in sport. British Journal of Sports Medicine. 39(6):319-323. https://doi.org/10.1136/bjsm.2005.018549

34. PETERSEN, J.; THORBORG, K.; NIELSEN, M.; BUDTZ-JORGENSEN, E.; HÖLMICH, P. 2011. Preventive effect of eccentric training on acute hamstring injuries in Men's soccer: A clusterrandomized controlled trial. American Journal of Sports Medicine. 39(11):2296-2303. https://doi.org/10.1177/0363546511419277

35. RAYA-GONZÁLEZ, J.; SUÁREZ-ARRONES, L.; MORENO-PUENTEDURA, M.; RUÍZMÁRQUEZ, J.; SÁEZ DE VILLAREAL, E. 2017. Efectos en el rendimiento físico a corto plazo de dos programas de entrenamiento neuromuscular con diferente orientación aplicados en jugadores de fútbol de élite U-17. Revista Internacional de Ciencias Del Deporte. 13(48):88-103.

36. REILLY, T.; BANGSBO, J.; FRANKS, A. 2000. Anthropometric and physiological predispositions for elite soccer. Journal of Sports Sciences. 18(9):669-683. https:// doi.org/10.1080/02640410050120050

37. SCHACHE, A. 2012. Eccentric hamstring muscle training can prevent hamstring injuries in soccer players. Journal of Physiotherapy. 58(1):58. https://doi. org/10.1016/s1836-9553(12)70074-7

38. SCHUERMANS, J.; VAN TIGGELEN, D.; DANNEELS, L.; WITVROUW, E. 2016. Susceptibility to Hamstring Injuries in Soccer: A Prospective Study Using Muscle Functional Magnetic Resonance Imaging. American Journal of Sports Medicine. 44(5):1276-1285. https:// doi.org/10.1177/0363546515626538

39. SERNER, A.; MOSLER, A.; TOL, J.; BAHR, R.; WEIR, A. 2019. Mechanisms of acute adductor longus injuries in male football players: A systematic visual video analysis. British Journal of Sports Medicine. 53(3):158-164.

40. SEYMORE, K.; DOMIRE, Z.; DEVITA, P.; RIDER, P.; KULAS, A. 2017. The effect of Nordic hamstring strength training on muscle architecture, stiffness, and strength. European Journal of Applied Physiology. 117(5):943-953. https://doi.org/10.1007/s00421-017-3583-3

41. SIDDLE, J.; GREIG, M.; WEAVER, K.; PAGE, R.; HARPER, D.; BROGDEN, C. 2019. Acute adaptations and subsequent preservation of strength and speed measures following a Nordic hamstring curl intervention: a randomised controlled trial. Journal of Sports Sciences. 37(8):911-920. https:// doi.org/10.1080/02640414.2018.1535786

42. SUN, Y.; WEI, S.; ZHONG, Y.; FU, W.; LI.; L.; LIU, Y. 2015. How joint torques affect hamstring injury risk in sprinting swing-stance transition. Medicine and Science in Sports and Exercise. 47(2):373-380. https://dx.doi. org/10.1249/MSS.0000000000000404

43. THELEN, D.; CHUMANOV, E.; HOERTH, D.; BEST, T.; SWANSON, S.; HEIDERSCHEIT, B. 2005. Hamstring muscle kinematics during treadmill sprinting. Medicine and Science in Sports and Exercise. 37(1):108-114. https://doi. org/10.1249/01.mss.0000150078.79120.c8

44. TØNNESEEN, E.; HALFAWI, S.; HAI, S.; AUGEN, T.; NOKSEN, E. 2011. The effect on 40M repeated sprint training on maximum Sprinting Speed, Repeated Sprint speed endurance, vertical jump and aerobic capacity in young elite male soccer players. The Journal of Strength \& Conditioning Research. 25(9):2364-2370. https://doi. org/10.1519/jsc.0b013e3182023a 65

45. VAN DER HORST, N.; SMITTS, D.; PETERSEN, J.; GOEDHART, E.; BACKX, F. 2015. The Preventive Effect of the Nordic Hamstring Exercise on Hamstring Injuries in Amateur Soccer Players: A Randomized Controlled Trial. American Journal of Sports Medicine. 43(6):1316-1323. https://doi.org/10.1177/0363546515574057 
46. WOODS, C.; HAWKINS, R.; MALTBY, S.; HULSE, M.; THOMAS, A.; HODSON, A. 2004. The Football Association Medical Research Programme: An audit of injuries in professional football - Analysis of hamstring injuries. British Journal of Sports Medicine. 38(1):36-41. https://doi.org/10.1136/bjsm.2002.002352
47. YU, B.; QUEEN, R.; ABBEY, A.; LIU, Y.; MOORMAN, C.; GARRETT, W. 2008. Hamstring muscle kinematics and activation during overground sprinting. Journal of Biomechanics. 41(15):3121-3126. https:// doi.org/10.1016/j.jbiomech.2008.09.005 